Pacific

Journal of

Mathematics

\title{
COMPOSITION OPERATORS ON STRICTLY PSEUDOCONVEX DOMAINS WITH SMOOTH SYMBOL
}

HyUnGWOON KoO AND Song-YING LI 


\title{
COMPOSITION OPERATORS ON STRICTLY PSEUDOCONVEX DOMAINS WITH SMOOTH SYMBOL
}

\author{
HYUNGWOON KoO AND SONG-YING LI
}

\begin{abstract}
It is well known that the composition operator $C_{\phi}$ is unbounded on Hardy and Bergman spaces on the unit ball $B_{n}$ in $\mathbb{C}^{n}$ when $n>1$ for a linear holomorphic self-map $\phi$ of $B_{n}$. We find a sufficient and necessary condition for a composition operator with smooth symbol to be bounded on Hardy or Bergman spaces over a bounded strictly pseudoconvex domain in $\mathbb{C}^{n}$. Moreover, we show that this condition is equivalent to the compactness of the composition operator from a Hardy or Bergman space into the Bergman space whose weight is $\frac{1}{4}$ bigger. We also prove that a certain jump phenomenon occurs when the composition operator is not bounded. Our results generalize known results on the unit ball to strictly pseudoconvex domains.
\end{abstract}

\section{Introduction}

Let $D$ be a bounded strictly pseudoconvex domain in $\mathbb{C}^{n}$ with a smooth boundary and let $d(z)$ be the distance from $z \in D$ to $\partial D$. Let $H(D)$ be the set of all holomorphic functions on $D$. For $0<p<\infty$ and $\alpha>-1$, the weighted Bergman space $A_{\alpha}^{p}(D)$ is the space of all $f \in H(D)$ for which

$$
\|f\|_{A_{\alpha}^{p}}^{p}=\int_{D}|f(z)|^{p} d V_{\alpha}(z)<\infty,
$$

where $d V_{\alpha}(z)=d(z)^{\alpha} d V(z)$ and $d V$ is the Lebesgue measure on $D$. Also, for $0<p<\infty$, the Hardy space $H^{p}(D)$ is the space of all $f \in H(D)$ for which

$$
\|f\|_{H^{p}}^{p}=\lim _{\epsilon \rightarrow 0} \int_{\partial D_{\epsilon}}|f(\zeta)|^{p} d \sigma_{\epsilon}(\zeta)<\infty,
$$

where $\sigma_{\epsilon}$ is the surface measure on $\partial D_{\epsilon}=\{z \in D: d(z)=\epsilon\}$. It is well known

H. Koo was supported by NRF of Korea (2012R1A1A2000705). Li was partially supported by the Minjing Scholar Fund from Fujian Normal University, Fujian, China.

MSC2010: primary 47B33; secondary 32T15, 32A36.

Keywords: composition operator, strictly pseudoconvex domain, boundedness, smooth symbol. 
(see [Krantz 2001]) that the admissible limit $f^{*}(\zeta)$ exists for almost every $\zeta \in \partial D$ when $f \in H^{p}(D)$ and

$$
\|f\|_{H^{p}}^{p}=\int_{\partial D}\left|f^{*}(\zeta)\right|^{p} d \sigma_{\epsilon}(\zeta)<\infty
$$

where $\sigma$ is the surface area measure on $\partial D$. For notational convenience we may view $H^{p}(D)$ as $A_{-1}^{p}(D)$.

Let $\phi=\left(\phi_{1}, \ldots, \phi_{n}\right): D \rightarrow D$ be a holomorphic self-map on $D$. Then $\phi$ induces the composition operator, $C_{\phi}$, defined on $H(D)$ by

$$
C_{\phi}(f)=f \circ \phi .
$$

When $D$ is the unit disk, $\Delta$, in $\mathbb{C}$, every composition operator is bounded on the weighted Bergman spaces and the Hardy spaces by Littlewood's subordination principle. On the other hand, when $D$ is the unit ball, $B_{n}$, in $\mathbb{C}^{n}$ with $n \geq 2$, it is known that not every composition is bounded on the weighted Bergman spaces or the Hardy spaces. Among the early examples of unbounded composition operators on $H^{p}\left(B_{2}\right)$, the example $\phi\left(z_{1}, z_{2}\right)=\left(2 z_{1} z_{2}, 0\right)$ is due to J.H. Shapiro and the examples $\phi\left(z_{1}, z_{2}\right)=\left(\psi\left(z_{1}, z_{2}\right), 0\right)$ for $\psi$ inner were given by MacCluer [1984] and Cima, Stanton, and Wogen [Cima et al. 1984]. Other than the Carleson measure characterization there is no satisfactory criteria known for general symbols up to present time. Since a holomorphic linear map $\phi$ can not guarantee $C_{\phi}$ is bounded on Hardy and Bergman spaces when $n>1$, one may concentrate on finding a good criteria for smooth holomorphic $\phi \in C^{\infty}\left(\bar{B}_{n}\right)$ so that $C_{\phi}$ is bounded on Hardy spaces, $H^{2}\left(B_{n}\right)$, and Bergman spaces, $A^{2}\left(B_{n}\right)$.

When $\phi$ is smooth up to the boundary, Warren Wogen [1988] found a necessary and sufficient condition for $C_{\phi}$ to be bounded on $H^{p}\left(B_{n}\right)$. This was generalized to $A_{\alpha}^{p}\left(B_{n}\right)$ in [Koo and Smith 2007], where the authors also showed what is called the jump phenomenon: if $\phi$ is smooth up to the boundary and $C_{\phi}$ is not bounded on $A_{\alpha}^{p}\left(B_{n}\right)$, then $C_{\phi}: A_{\alpha}^{p}\left(B_{n}\right) \nrightarrow \rightarrow A_{\alpha-\epsilon}^{p}\left(B_{n}\right)$ for all $0 \leq \epsilon<\frac{1}{4}$. It was also proved [Koo and Park 2010] that the boundedness of $C_{\phi}: A_{\alpha}^{p}\left(B_{n}\right) \rightarrow A_{\alpha}^{p}\left(B_{n}\right)$ is equivalent to the compactness of $C_{\phi}: A_{\alpha}^{p}\left(B_{n}\right) \rightarrow A_{\alpha+1 / 4}^{p}\left(B_{n}\right)$ when $\phi$ is smooth up to the boundary. Wogen's original proof [1988] is quite long and involves various local analyses of the inducing map. Koo and Wang [2010] gave a much simpler proof of Wogen's result using certain compactness argument.

In this paper, we generalize the boundedness criteria and the jump phenomenon of composition operators with smooth symbols to bounded strictly pseudoconvex domains in $\mathbb{C}^{n}$. We adapt the compactness argument of [Koo and Wang 2010] in our proof. Our main theorem is the following, with $Q_{\phi}(\zeta)$ defined as in (3-1).

Theorem 1.1. Let $0<p<\infty$ and $\alpha \geq-1$. Let $\phi: D \rightarrow D$ be a holomorphic map with $\phi \in C^{4}(\bar{D})$. Then the following are equivalent. 
(1) $C_{\phi}: A_{\alpha}^{p}(D) \rightarrow A_{\alpha}^{p}(D)$ is bounded.

(2) $C_{\phi}: A_{\alpha}^{p}(D) \rightarrow A_{\alpha+1 / 4}^{p}(D)$ is compact.

(3) $Q_{\phi}(\zeta)<1$ on $\phi^{-1}(\partial D)$.

Moreover, if $C_{\phi}: A_{\alpha}^{p}(D) \nrightarrow \rightarrow A_{\alpha}^{p}(D)$, then $C_{\phi}: A_{\alpha}^{p}(D) \nrightarrow \rightarrow A_{\alpha+\epsilon}^{p}(D)$ for all $0<\epsilon<\frac{1}{4}$.

Remark. For $\phi(z)=\left(z_{1}+z_{2}^{2} / 2,0\right): B_{2} \rightarrow B_{2}$, we know $C_{\phi}: A_{\alpha}^{p}\left(B_{2}\right) \rightarrow A_{\alpha+1 / 4}^{p}\left(B_{2}\right)$ is bounded [Koo and Smith 2007] but not compact [Koo and Park 2010].

In Section 2, we review well-known facts on strictly pseudoconvex domains $D$ and Wogen's result on the unit ball. In Section 3, we study local behavior of maps on $D$ which are smooth on $\bar{D}$, especially holomorphic self-maps of $D$. We prove our main theorem in Section 4.

Throughout the paper we use the same letter $C$ to denote various positive constants which may vary at each occurrence but do not depend on the essential parameters. Variables indicating the dependency of constants $C$ will be often specified in parentheses. For nonnegative quantities $X$ and $Y$ the notation $X \lesssim Y$ or $Y \gtrsim X$ means $X \leq C Y$ for some inessential constant $C$. Similarly, we write $X \approx Y$ if both $X \lesssim Y$ and $Y \lesssim X$ hold.

\section{Background}

Strictly pseudoconvex domain. A $C^{2}$-domain $D \subset \mathbb{C}^{n}$ is strictly pseudoconvex if there is a defining function $r \in C^{2}\left(\mathbb{C}^{n}\right)$ such that

$$
D=\left\{z \in \mathbb{C}^{n}: r(z)>0\right\}
$$

and there exists $C>0$ such that

$$
C|w|^{2} \leq-\sum_{j=1}^{n} \frac{\partial^{2} r(\zeta)}{\partial \zeta_{i} \partial \bar{\zeta}_{j}} w_{i} \bar{w}_{j}
$$

for all $\zeta \in \partial D$ and for all $w \in \mathbb{C}^{n}$. For $\epsilon>0$, let

$$
D_{\epsilon}=\{z \in D: r(z)>\epsilon\} .
$$

For $z, w \in \bar{D}$, define a quasimetric $d(z, w)$ by

$$
d(z, w)=r(z)+r(w)+\left|\sum_{j=1}^{n} \frac{\partial r(w)}{\partial w_{j}}\left(z_{j}-w_{j}\right)\right|+|z-w|^{2} .
$$

For $z, w \in \bar{D}$, let

$$
X(z, w)=r(w)+\sum_{j=1}^{n} \frac{\partial r(w)}{\partial w_{j}}\left(z_{j}-w_{j}\right)+\frac{1}{2} \sum_{j, k=1}^{n} \frac{\partial^{2} r(w)}{\partial w_{i} \partial w_{j}}\left(z_{j}-w_{j}\right)\left(z_{k}-w_{k}\right) .
$$


Note that, by Taylor expansion of $r$ near $w$, we get $r(z)=-r(w)+2 \operatorname{Re} X(z, w)+\sum_{i, j=1}^{n} \frac{\partial^{2} r(w)}{\partial w_{i} \partial \bar{w}_{j}}\left(z_{i}-w_{i}\right)\left(\bar{z}_{j}-\bar{w}_{j}\right)+O\left(|z-w|^{3}\right)$. Thus, when $D$ is strictly pseudoconvex and $z \in \bar{D}$ is near $\eta \in \partial D$,

$$
\operatorname{Re} X(z, \eta) \geq 0
$$

by (2-1). Moreover, it is well known from work of C. Fefferman [1974] that there exists $\delta_{D}>0$ such that

$$
|X(z, w)| \approx d(z, w)
$$

for all $(z, w) \in R_{\delta_{D}}$, where

$$
R_{\delta}=\{(z, w) \in \bar{D} \times \bar{D}: r(z)+r(w)+|z-w|<\delta\} .
$$

Carleson measures. For any $\zeta \in \partial D$, we can define a Carleson region centered at $\zeta$ with radius $\delta$ by

$$
\mathscr{C}(\zeta, \delta)=\{z \in D: d(z, \zeta)<\delta\}
$$

A positive Borel measure $\mu$ on $\bar{D}$ is said to be a Carleson measure if there is a constant $M>0$ such that, for all $\zeta \in \partial D$ and $\delta>0$,

$$
\mu(\overline{\mathscr{C}(\zeta, \delta)}) \leq M \sigma(\overline{\mathscr{C}(\zeta, \delta)} \cap \partial D),
$$

and such a measure $\mu$ is said to be a vanishing Carleson measure if

$$
\lim _{\delta \rightarrow 0} \sup _{\zeta \in \partial D} \frac{\mu(\overline{\mathscr{C}(\zeta, \delta)})}{\sigma(\overline{\mathscr{C}(\zeta, \delta)} \cap \partial D)}=0 .
$$

Also, for $\alpha>-1$, a positive Borel measure $\mu$ on $D$ is said to be an $\alpha$-Carleson measure if there is a constant $M>0$ such that, for all $\zeta \in \partial D$ and $\delta>0$,

$$
\mu(\mathscr{C}(\zeta, \delta)) \leq M V_{\alpha}(\mathscr{C}(\zeta, \delta)),
$$

and such a measure $\mu$ is said to be a vanishing $\alpha$-Carleson measure if

$$
\lim _{\delta \rightarrow 0} \sup _{\zeta \in \partial D} \frac{\mu(\mathscr{C}(\zeta, \delta))}{V_{\alpha}(\mathscr{C}(\zeta, \delta))}=0 .
$$

By [Krantz and Li 1994] the $V_{\alpha}$-volume of $\mathscr{C}(\zeta, \delta)$ and the surface area of the intersection $\overline{\mathscr{C}(\zeta, \delta)} \cap \partial D$ are

$$
V_{\alpha}(\mathscr{C}(\zeta, \delta)) \approx \delta^{n+1+\alpha} \quad \text { and } \quad \sigma(\overline{\mathscr{C}(\zeta, \delta)} \cap \partial D) \approx \delta^{n},
$$

respectively. 
The next theorem follows from Hörmander's work [1967] on Carleson measures, the work on Bergman and Szegó kernels by Fefferman [1974] and Phong and Stein [1977], together with Krantz and Li's [1994; 1995a; 1995b] work on Hardy spaces and Bergman spaces.

Theorem 2.1. Let $D$ be a smooth bounded strictly pseudoconvex domain in $\mathbb{C}^{n}$, $0<p<\infty$ and $\alpha>-1$. Let $\mu$ be a positive Borel measure on $\bar{D}$ and $v$ a positive Borel measure on D.

(1) The inclusion $H^{p}(D) \hookrightarrow L^{p}(\mu)$ is continuous if and only if $\mu$ is a Carleson measure, and compact if and only if $\mu$ is a vanishing Carleson measure.

(2) The inclusion $A_{\alpha}^{p}(D) \hookrightarrow L^{p}(v)$ is continuous if and only if $v$ is an $\alpha$-Carleson measure, and compact if and only if $\mu$ is a vanishing $\alpha$-Carleson measure.

Let $\phi: D \rightarrow D$ be a holomorphic mapping and, for a holomorphic function $f$ on $D$, let

$$
C_{\phi}(f)(z)=f \circ \phi(z) .
$$

Since $D$ is bounded, $\phi$ has admissible limit $\phi^{*}(\zeta)$ almost everywhere in $\partial D$. So, when $\xi \in \partial D$, we define $\phi(\xi)=: \phi^{*}(\xi)$. Let $\sigma \circ \phi^{-1}$ and $V_{\alpha} \circ \phi^{-1}$ be the measures on $\bar{D}$ and $D$ defined by

for all $E \subset \bar{D}$ and

$$
\sigma \circ \phi^{-1}(E)=\int_{\phi^{*-1}(E)} d \sigma(\zeta)
$$

$$
V_{\alpha} \circ \phi^{-1}(E)=\int_{\phi^{-1}(E)} d V_{\alpha}(z)
$$

for all $E \subset D$, respectively. Then, by a change of variables, we have

and

$$
\int_{\partial D}\left|C_{\phi} f(\zeta)\right|^{p} d \sigma(\zeta)=\int_{\bar{D}}|f(z)|^{p} d \sigma \circ \phi^{-1}(z)
$$

$$
\int_{D}\left|C_{\phi} f(z)\right|^{p} d V_{\alpha}(z)=\int_{D}|f(z)|^{p} d V_{\alpha} \circ \phi^{-1}(z) .
$$

Therefore, as a corollary of Theorem 2.1 we have the following characterization.

Corollary 2.2. Let $0<p<\infty, \alpha, \beta>-1$, and $\phi: D \rightarrow D$ be a holomorphic mapping.

(1) $C_{\phi}: H^{p}(D) \rightarrow H^{p}(D)$ is bounded if and only if $\sigma \circ \phi^{-1}$ is a Carleson measure, and compact if and only if $\sigma \circ \phi^{-1}$ is a vanishing Carleson measure.

(2) $C_{\phi}: H^{p}(D) \rightarrow A_{\alpha}^{p}(D)$ is bounded if and only if $V_{\alpha} \circ \phi^{-1}$ is a Carleson measure, and compact if and only if $V_{\alpha} \circ \phi^{-1}$ is a vanishing Carleson measure.

(3) $C_{\phi}: A_{\alpha}^{p}(D) \rightarrow A_{\beta}^{p}(D)$ bounded if and only if $V_{\beta} \circ \phi^{-1}$ is an $\alpha$-Carleson measure, and compact if and only if $V_{\beta} \circ \phi^{-1}$ is a vanishing $\alpha$-Carleson measure. 
Wogen's theorem. Let $\phi: B_{n} \rightarrow B_{n}$ be holomorphic and $\phi \in C^{4}\left(\bar{B}_{n}\right)$. Then Wogen proved [1988] the following characterization for $C_{\phi}$ to be bounded in $H^{2}\left(B_{n}\right)$, which was generalized by Koo and Smith to $A_{\alpha}^{p}\left(B_{n}\right)$ [2007], and by Koo and Park to holomorphic Sobolev spaces [2010]. For $z, \zeta \in \mathbb{C}^{n}$ and a smooth function $g$, let

$$
\mathscr{D}_{\zeta} g(z)=\sum_{j=1}^{n} \zeta_{j} \frac{\partial g}{\partial z_{j}}(z) \text { and } \mathscr{D}_{\bar{\zeta}} g(z)=\sum_{j=1}^{n} \bar{\zeta}_{j} \frac{\partial g}{\partial \bar{z}_{j}}(z) \text {. }
$$

For $z, w \in \mathscr{C}^{n}$, let $\langle z, w\rangle$ be the Hermitian inner product defined by

$$
\langle z, w\rangle=\sum_{j=1}^{n} z_{j} \bar{w}_{j}
$$

Theorem 2.3. Let $\phi: B_{n} \rightarrow B_{n}$ be holomorphic and $\phi \in C^{4}\left(\bar{B}_{n}\right)$. Let $0<p<\infty$, $\alpha \geq-1$. For $\eta \in \partial B_{n}$, let $H_{\eta}(z)=\langle\phi(z), \eta\rangle$. Then $C_{\phi}: A_{\alpha}^{p}\left(B_{n}\right) \rightarrow A_{\alpha}^{p}\left(B_{n}\right)$ is bounded if and only if

$$
\left|\mathscr{D}_{\tau \tau} H_{\eta}(\zeta)\right|<\mathscr{D}_{\zeta} H_{\eta}(\zeta)
$$

for all $\zeta, \eta, \tau \in \partial B_{n}$ such that

$$
\zeta \in \phi^{-1}\left(\partial B_{n}\right), \quad \eta=\phi(\zeta), \quad\langle\zeta, \tau\rangle=0 .
$$

Koo and Smith [2007] proved that the following jump phenomenon occurs when $C_{\phi}$ is not bounded.

Theorem 2.4. Let $\phi: B_{n} \rightarrow B_{n}$ be holomorphic and $\phi \in C^{4}\left(\bar{B}_{n}\right)$. Let $0<p<\infty$, $\alpha \geq-1$. If $C_{\phi}$ is not bounded on $A_{\alpha}^{p}\left(B_{n}\right)$, then $C_{\phi}: A_{\alpha}^{p}\left(B_{n}\right) \not \rightarrow A_{\alpha+\epsilon}^{p}\left(B_{n}\right)$ for all $0 \leq \epsilon<\frac{1}{4}$.

The following was proved for the critical index $\epsilon=\frac{1}{4}$ [Koo and Park 2010].

Theorem 2.5. Let $\phi: B_{n} \rightarrow B_{n}$ be holomorphic and $\phi \in C^{4}\left(\bar{B}_{n}\right)$. Let $0<p<\infty$ and $\alpha \geq-1$. Then $C_{\phi}: A_{\alpha}^{p}\left(B_{n}\right) \rightarrow A_{\alpha}^{p}\left(B_{n}\right)$ is bounded if and only if $C_{\phi}: A_{\alpha}^{p}\left(B_{n}\right) \rightarrow$ $A_{\alpha+1 / 4}^{p}\left(B_{n}\right)$ is compact.

\section{Local estimates of smooth holomorphic maps on $D$}

Throughout this section we assume that $\phi: D \rightarrow D$ is a holomorphic mapping with $\phi \in C^{4}(\bar{D})$ where $D$ is a bounded strictly pseudoconvex domain with a smooth boundary. For $z \in \mathbb{C}^{n}$, we use the following notation:

$$
z=\left(z_{1}, z_{2}, \ldots, z_{n}\right)=\left(z_{1}, z^{\prime}\right)=\left(z_{1}, z_{2}, z^{\prime \prime}\right), \quad z_{j}=x_{j}+i y_{j}(1 \leq j \leq n) .
$$

For $w$ near $\partial D$, let

$$
v(w)=|\partial r(w)|^{-1} \partial r(w)
$$


where

$$
\partial r(z)=\left(\frac{\partial r(z)}{\partial z_{1}}, \ldots, \frac{\partial r(z)}{\partial z_{n}}\right)
$$

For $\eta \in \partial D$, let

$$
\phi_{\eta}(z)=X(\phi(z), \eta)
$$

and let

$$
Q_{\phi}(\zeta, \eta)=\sup _{\tau}\left\{\left|\frac{\mathscr{D}_{\tau \tau}^{2} \phi_{\eta}(\zeta)}{\mathscr{D}_{\nu(\zeta)} \phi_{\eta}(\zeta)}-\frac{\mathscr{D}_{\tau \tau}^{2} r(\zeta)}{|\partial r(\zeta)|}\right| \cdot \frac{|\partial r(\zeta)|}{\left|\mathscr{D}_{\tau \bar{\tau}}^{2} r(\zeta)\right|}:\langle\tau, \nu(\zeta)\rangle=0\right\} .
$$

If $\eta=\phi(\zeta)$, we let

$$
Q_{\phi}(\zeta)=Q_{\phi}(\zeta, \phi(\zeta))
$$

For $D=B_{n}$, it is easy to check that $\phi_{\eta}=2 H_{\eta}-2$ and the condition on Theorem 2.3 is equivalent to $Q_{\phi}(\zeta)<1$ for all $\zeta \in \phi^{-1}(\partial D)$.

Proposition 3.1. Let $\zeta \in \partial D$ and $\eta=\phi(\zeta) \in \partial D$. Then

(1) $\mathscr{D}_{\mathcal{V}(\zeta)} \phi_{\eta}(\zeta)>0$

(2) $\mathscr{D}_{\tau} \phi_{\eta}(\zeta)=0$ for all $\tau$ with $\langle v(\zeta), \tau\rangle=0$,

(3) $Q_{\phi}(\zeta) \leq 1$.

Proof. Let $\zeta, \eta \in \partial D$, and $\langle v(\zeta), \tau\rangle=0$. Without loss of generality, we may choose local coordinates near $(\zeta, \eta) \in \partial D \times \partial D \subset \mathbb{C}^{2 n}$ such that

$$
\zeta=\eta=(0, \ldots, 0), \quad v(\zeta)=v(\eta)=(1,0, \ldots, 0), \quad \tau=(0,1,0, \ldots, 0) .
$$

For $1 \leq i, j \leq n$, let

$$
r_{i}=\frac{\partial r(\zeta)}{\partial z_{i}}, \quad r_{i j}=\frac{\partial^{2} r(\zeta)}{\partial z_{i} \partial z_{j}}, \quad r_{i \bar{j}}=\frac{\partial^{2} r(\zeta)}{\partial z_{i} \partial \bar{z}_{j}},
$$

and let

$$
a_{i}=\frac{\partial r(\eta)}{\partial z_{i}}, \quad a_{i j}=\frac{\partial^{2} r(\eta)}{\partial z_{i} \partial z_{j}} .
$$

Also, for $1 \leq i, j, \ell \leq n$, let

$$
b_{i}^{\ell}=\frac{\partial \phi_{\ell}(\zeta)}{\partial z_{i}}, \quad b_{i j}^{\ell}=\frac{\partial^{2} \phi_{\ell}(\zeta)}{\partial z_{i} \partial z_{j}} .
$$

From the definition of $X$, we have

$$
\begin{aligned}
\phi_{\eta}(z) & =: X(\phi(z), \eta) \\
& =\sum_{j=1}^{n} \frac{\partial r(\eta)}{\partial \eta_{j}}\left(\phi_{j}(z)-\eta_{j}\right)+\frac{1}{2} \sum_{i, j=1}^{n} \frac{\partial^{2} r(\eta)}{\partial \eta_{i} \partial \eta_{j}}\left(\phi_{i}(z)-\eta_{i}\right)\left(\phi_{j}(z)-\eta_{j}\right),
\end{aligned}
$$


and thus

$$
\phi_{\eta}(z)=a_{1} \phi_{1}(z)+\frac{1}{2} \sum_{i, j=1}^{n} a_{i j} \phi_{i}(z) \phi_{j}(z) .
$$

Since the harmonic function $\operatorname{Re} \phi_{1}$ takes a minimum at $\zeta$ and $v(\zeta)$ is the inward normal vector at $\zeta \in \partial D$, by Hopf's lemma, we have

$$
b_{1}^{1}=\frac{\partial \phi_{1}(\zeta)}{\partial \zeta_{1}}=\frac{\partial \operatorname{Re} \phi_{1}}{\partial x_{1}}(\zeta)>0
$$

Since $v(\zeta)=(1,0, \ldots, 0)$, for $z$ near $\zeta$

$$
r(z)=2 r_{1} x_{1}+O\left(|z|^{2}\right) \quad\left(r_{1}>0\right) .
$$

Therefore, there are $\epsilon, \delta>0$ such that

$$
z=\left(x_{1}, z^{\prime}\right) \in D \quad \text { if } 0<x_{1} \leq \delta \quad \text { and } \quad\left|z^{\prime}\right|^{2}=\epsilon\left|x_{1}\right|
$$

Then, for all $\left(x_{1}, z^{\prime}\right)$ with $0<x_{1} \leq \delta$ and $\left|z^{\prime}\right|^{2}=\epsilon\left|z_{1}\right|$, we have

$$
0 \leq \operatorname{Re} \phi_{1}\left(x_{1}, z^{\prime}\right)=\operatorname{Re}\left(b_{1}^{1} x_{1}+\sum_{j=2}^{n} b_{j}^{1} z_{j}\right)+O\left(|z|^{2}\right) .
$$

From this, we can easily deduce that

$$
b_{j}^{1}=\frac{\partial \phi_{1}(\zeta)}{\partial \zeta_{j}}=0 \quad(2 \leq j \leq n) .
$$

Then, from (3-2), (3-3), and (3-4), we have

$$
\begin{aligned}
\phi_{\eta}(z) & =a_{1}\left(b_{1}^{1} z_{1}+\frac{1}{2} \sum_{i, j=1}^{n} b_{i j}^{1} z_{i} z_{j}\right)+\frac{1}{2} \sum_{k, \ell=1}^{n}\left(\sum_{i, j=1}^{n} a_{i j} b_{k}^{i} b_{\ell}^{j}\right) z_{k} z_{\ell}+O\left(|z|^{3}\right) \\
& =a_{1} b_{1}^{1}\left[z_{1}+\frac{1}{2 a_{1} b_{1}^{1}} \sum_{i, j=1}^{n}\left[a_{1} b_{i j}^{1}+\sum_{k, \ell=1}^{n} a_{k \ell} b_{i}^{k} b_{j}^{\ell}\right] z_{i} z_{j}\right]+O\left(|z|^{3}\right)
\end{aligned}
$$

From this we easily conclude (1) and (2).

For (3), let

$$
c_{i j}=\frac{r_{1}}{2 a_{1} b_{1}^{1}}\left[a_{1} b_{i j}^{1}+\sum_{k, \ell=1}^{n} a_{k \ell} b_{i}^{k} b_{j}^{\ell}\right]-\frac{r_{i j}}{2} .
$$


Then we get

$$
\begin{array}{r}
\phi_{\eta}(z)=\frac{a_{1} b_{1}^{1}}{r_{1}}\left[r_{1} z_{1}+\frac{1}{2} \sum_{i, j=1}^{n} r_{i j} z_{i} z_{j}+\frac{1}{2} \sum_{i, j=1}^{n} r_{i} z_{i} \bar{z}_{j}\right] \\
+\frac{a_{1} b_{1}^{1}}{r_{1}}\left[\sum_{i, j=1}^{n} c_{i j} z_{i} z_{j}-\frac{1}{2} \sum_{i, j=1}^{n} r_{i j} z_{i} \bar{z}_{j}\right]+O\left(|z|^{3}\right) .
\end{array}
$$

Note that, for $z$ near $\zeta$,

$$
r(z)=2 \operatorname{Re}\left(r_{1} z_{1}+\frac{1}{2} \sum_{i, j=1}^{n} r_{i j} z_{i} z_{j}+\frac{1}{2} \sum_{i, j=1}^{n} r_{i} z_{i} \bar{z}_{j}\right)+O\left(|z|^{3}\right) .
$$

Now consider a point $\left(s, t e^{i \theta}, 0^{\prime \prime}\right)$ near $\zeta$, with $s, t \geq 0$. (Here and below, $0^{\prime \prime}$ stands for the origin in $\mathscr{C}^{n-2}$; see start of Section 3.) We have

$$
r\left(s, t e^{i \theta}, 0^{\prime \prime}\right)=2 r_{1} s+\left(\operatorname{Re}\left(r_{22} e^{2 i \theta}\right)+r_{22}\right) t^{2}+O\left(s^{2}+s t+t^{3}\right),
$$

and thus

$$
r\left(s, t e^{i \theta}, 0^{\prime \prime}\right) \approx t^{5 / 2} \text { if } s=t^{5 / 2}-\frac{1}{2 r_{1}}\left(\operatorname{Re}\left(r_{22} e^{2 i \theta}\right)+r_{2 \overline{2}}\right) t^{2} .
$$

Then, with $z:=\left(s, t e^{i \theta}, 0^{\prime \prime}\right)$, by (2-3) and (3-6), we have

$$
\begin{aligned}
0 \leq \operatorname{Re} \phi_{\eta}(z) & =\frac{a_{1} b_{1}^{1}}{2 r_{1}} r(z)+\frac{a_{1} b_{1}^{1}}{r_{1}} \operatorname{Re}\left(c_{22} t^{2} e^{2 i \theta}-\frac{1}{2} r_{22} t^{2}\right)+O\left(t^{3}\right) \\
& =\frac{a_{1} b_{1}^{1}}{r_{1}} \operatorname{Re}\left(c_{22} e^{2 i \theta}-\frac{1}{2} r_{2 \overline{2}}\right) t^{2}+O\left(t^{5 / 2}\right)
\end{aligned}
$$

for all $\theta$. Thus

$$
\operatorname{Re}\left(c_{22} e^{2 i \theta}-\frac{1}{2} r_{22}\right) \geq 0, \quad \theta \in[0,2 \pi] .
$$

This implies

$$
\left|c_{22}\right| \leq-\frac{r_{2 \overline{2}}}{2}
$$

Since $v(\zeta)=(1,0, \ldots, 0)$ and $\tau=(0,1,0, \ldots, 0)$, by $(3-6)$ we have

$$
c_{22}=r_{1} \frac{1}{2} \frac{\partial^{2} \phi_{\eta}(\zeta)}{\partial \zeta_{2} \partial \zeta_{2}}\left(\frac{\partial \phi_{\eta}(\zeta)}{\partial \zeta_{1}}\right)^{-1}-\frac{r_{22}}{2}=\frac{|\partial r(\zeta)|}{2}\left(\frac{\mathscr{D}_{\tau \tau}^{2} \phi_{\eta}(\zeta)}{\mathscr{D}_{\mathcal{V}(\zeta)} \phi_{\eta}(\zeta)}-\frac{\mathscr{D}_{\tau \tau}^{2} r(\zeta)}{|\partial r(\zeta)|}\right)
$$

Therefore, we have

$$
\frac{|\partial r(\zeta)|}{2}\left|\frac{\mathscr{D}_{\tau \tau}^{2} \phi_{\eta}(\zeta)}{\mathscr{D}_{\nu(\zeta)} \phi_{\eta}(\zeta)}-\frac{\mathscr{D}_{\tau \tau}^{2} r(\zeta)}{|\partial r(\zeta)|}\right|=\left|c_{22}\right| \leq-\frac{1}{2} \frac{\partial^{2} r(\zeta)}{\partial z_{2} \partial \bar{z}_{2}}=-\frac{1}{2} \mathscr{D}_{\tau \bar{\tau}}^{2} r(\zeta) .
$$


The following lemma is the key local estimate for the proof of (3) $\Longrightarrow$ (1) of Theorem 1.1. First we introduce some notation. For $\delta>0$, let

$$
\begin{aligned}
V_{\delta} & =\left\{\xi \in \partial D:|X(\xi, \zeta)|<\delta \text { for some } \zeta \in \phi^{-1}(\partial D)\right\}, \\
W_{\delta} & =\left\{\eta \in \partial D:|X(\eta, \phi(\zeta))|<\delta \text { for some } \zeta \in \phi^{-1}(\partial D)\right\}, \\
K & =\left\{(\zeta, \phi(\zeta)) \in \partial D \times \partial D: \zeta \in \phi^{-1}(\partial D)\right\}, \\
K_{\delta} & =\left\{(z, \eta) \in \bar{D} \times \partial D:|X(z, \zeta)|+|X(\phi(\zeta), \eta)|<\delta, \zeta \in \phi^{-1}(\partial D)\right\} .
\end{aligned}
$$

Lemma 3.2. Suppose $Q_{\phi}(\xi)<1$ on $\phi^{-1}(\partial D)$. Then there are $\delta>0$ and $C>1$ such that, for all $(z, \eta) \in K_{\delta}$,

$$
\frac{1}{C}(|X(\phi(\zeta), \eta)|+|X(z, \zeta)|) \leq|X(\phi(z), \eta)| \leq C(|X(\phi(\zeta), \eta)|+|X(z, \zeta)|),
$$

where the point $\zeta \in \partial D$ is defined by the relation

$$
\min \left\{|X(\phi(w), \eta)|: w \in \bar{O}_{z}\right\}=|X(\phi(\zeta), \eta)|
$$

and $O_{z}$ is the connected component of $\phi^{-1}(\mathscr{C}(\eta, \delta))$ containing $z$.

Proof. Since $\phi \in C^{2}(\bar{D})$, there are $\epsilon, \delta>0$ such that $Q_{\phi}(z, \eta) \leq 1-\epsilon$ for all $(z, \eta) \in K_{\delta}$. Fix $(z, \eta) \in K_{\delta}$ and let $\zeta$ be any point such that

$$
\min \{|X(\phi(w), \eta)|: w \in\}=|X(\phi(\zeta), \eta)| .
$$

Note that $\zeta \in \partial D$, since $\phi_{\eta}(w)=X(\phi(w), \eta)$ is an open map as a holomorphic function on $D$. Without loss of generality, we may choose local coordinates near $(\zeta, \eta) \in \partial D \times \partial D \subset \mathbb{C}^{2 n}$ as in the proof of Proposition 3.1 so that

$$
\zeta=\eta=(0, \ldots, 0), \quad v(\zeta)=v(\eta)=(1,0, \ldots, 0) .
$$

Then, by Taylor expansion of $\phi_{\eta}$ at $\zeta$, we have

$$
\phi_{\eta}(z)=\phi_{\eta}(\zeta)+\sum_{j=1}^{n} a_{j} z_{j}+\frac{1}{2} \sum_{i, j=2}^{n} a_{i j} z_{i} z_{j}+O\left(\left|z_{1}\right|^{2}+\left|z_{1}\right|\left|z^{\prime}\right|+\left|z^{\prime}\right|^{3}\right) .
$$

By Proposition 3.1(1), we have $\mathscr{D}_{v(\zeta)} \phi_{\eta}(\zeta)>0$ when $\eta=\phi(\zeta)$. Therefore, by shrinking $\delta$ if necessary, we may assume that $\mathscr{D}_{\nu(\zeta)} \phi_{\eta}(\zeta) \neq 0$ for all $(\zeta, \eta) \in K_{\delta}$, and thus

$$
a_{1}=\frac{\partial \phi_{\eta}}{\partial z_{1}}(\zeta)=\mathscr{D}_{\mathcal{v}(\zeta)} \phi_{\eta}(\zeta) \neq 0
$$

Since $\zeta$ is the local minimum point of $\left|\phi_{\eta}\right|$, by Taylor expansion of $\phi_{\eta}(z)$ at $\zeta$ with $z=\left(s, t e^{i \theta}, 0^{\prime \prime}\right)$ as in (3-7), we see that

$$
a_{j}=\frac{\partial \phi_{\eta}}{\partial z_{j}}(\zeta)=0 \quad \text { if } j \geq 2
$$


Thus we have

$$
\phi_{\eta}(z)=\phi_{\eta}(\zeta)+a_{1} z_{1}+\frac{1}{2} \sum_{i, j=2}^{n} a_{i j} z_{i} z_{j}+O\left(\left|z_{1}\right|^{2}+\left|z_{1}\right|\left|z^{\prime}\right|+\left|z^{\prime}\right|^{3}\right) .
$$

Note that by assumption we have $Q_{\phi}(\zeta, \eta) \leq 1-\epsilon$, since $(\zeta, \eta) \in K_{\delta}$. Define $F$ and $G$ on $\mathbb{C}^{n-1}$ by

$$
F\left(z^{\prime}\right)=\frac{1}{2} \sum_{i, j=2}^{n}\left(\frac{a_{i j}}{a_{1}}-\frac{r_{i j}}{r_{1}}\right) z_{i} z_{j}, \quad G\left(z^{\prime}\right)=-(1-\epsilon) \sum_{i, j=2}^{n} \frac{r_{i \bar{j}}}{r_{1}} z_{i} \bar{z}_{j} .
$$

Then the condition $Q_{\phi}(\zeta, \eta) \leq 1-\epsilon$ implies $\left|\mathscr{D}_{\tau^{\prime} \tau^{\prime}} F\right| \leq \mathscr{D}_{\tau^{\prime} \bar{\tau}^{\prime}} G$ for all $\tau^{\prime} \in \mathbb{C}^{n-1}$. But straightforward calculations show that

$$
\mathscr{D}_{\tau^{\prime} \tau^{\prime}} F\left(z^{\prime}\right)=2 F\left(\tau^{\prime}\right), \quad \mathscr{D}_{\tau^{\prime} \bar{\tau}^{\prime}} G\left(z^{\prime}\right)=G\left(\tau^{\prime}\right) .
$$

Therefore, we have

$$
\left|\sum_{i, j=2}^{n}\left(\frac{a_{i j}}{a_{1}}-\frac{r_{i j}}{r_{1}}\right) z_{i} z_{j}\right| \leq-(1-\epsilon) \sum_{i, j=2}^{n} \frac{r_{i} \bar{j}}{r_{1}} z_{i} \bar{z}_{j} .
$$

Since $D$ is strictly pseudoconvex, from this inequality together with (2-1), we have

$$
-\sum_{i, j=2}^{n} \frac{r_{i} \bar{j}}{r_{1}} z_{i} \bar{z}_{j}-\left|\sum_{i, j=2}^{n}\left(\frac{a_{i j}}{a_{1}}-\frac{r_{i j}}{r_{1}}\right) z_{i} z_{j}\right| \geq \epsilon C\left|z^{\prime}\right|^{2} .
$$

Therefore, by (3-9) we have

$$
\begin{aligned}
& \left|\operatorname{Re}\left(\phi_{\eta}(z)-\phi_{\eta}(\zeta)\right)\right| \\
& \geq\left|a_{1}\right| \operatorname{Re}\left(z_{1}+\frac{1}{2} \sum_{i, j=2}^{n} \frac{r_{i j}}{r_{1}} z_{i} z_{j}+\frac{1}{2} \sum_{i, j=2}^{n} \frac{r_{i \bar{j}}}{r_{1}} z_{i} \bar{z}_{j}\right) \\
& \quad-\left|a_{1}\right|\left(\frac{1}{2} \sum_{i, j=2}^{n} \frac{r_{i} \bar{j}}{r_{1}} z_{i} \bar{z}_{j}+\frac{1}{2}\left|\sum_{i, j=2}^{n}\left(\frac{a_{i j}}{a_{1}}-\frac{r_{i j}}{r_{1}}\right) z_{i} z_{j}\right|\right)+O\left(\left|z_{1}\right|^{2}+\left|z_{1}\right|\left|z^{\prime}\right|+\left|z^{\prime}\right|^{3}\right) \\
& \geq \frac{\left|a_{1}\right|}{2 r_{1}} r(z)+\left|a_{1}\right| \frac{\epsilon C\left|z^{\prime}\right|^{2}}{2}+O\left(\left|z_{1}\right|^{2}+\left|z_{1}\right|\left|z^{\prime}\right|+\left|z^{\prime}\right|^{3}\right) .
\end{aligned}
$$

Since $\left|\phi_{\eta}(z)-\phi_{\eta}(\zeta)\right| \lesssim\left|\phi_{\eta}(z)-\phi_{\eta}(\zeta)\right|+\left|\operatorname{Re}\left(\phi_{\eta}(z)-\phi_{\eta}(\zeta)\right)\right|$, by (3-9) we then have

$$
\left|\phi_{\eta}(z)-\phi_{\eta}(\zeta)\right| \gtrsim\left|a_{1} z_{1}+\frac{1}{2} \sum_{i, j=2}^{n} a_{i j} z_{i} z_{j}\right|+\left|z^{\prime}\right|^{2}+O\left(\left|z_{1}\right|^{2}+\left|z_{1}\right|\left|z^{\prime}\right|+\left|z^{\prime}\right|^{3}\right) .
$$


Since $|a+b|+c>|a| / M+(M c-|b|) / M$ for any $M \geq 1$, we see that there is $C>0$ such that

$$
\left|\phi_{\eta}(z)-\phi_{\eta}(\zeta)\right| \geq C\left(\left|z_{1}\right|+\left|z^{\prime}\right|^{2}\right)+O\left(\left|z_{1}\right|^{2}+\left|z_{1}\right|\left|z^{\prime}\right|+\left|z^{\prime}\right|^{3}\right) .
$$

Note that by (2-4) we have

$$
\begin{aligned}
|X(z, \zeta)| & \approx d(z, \zeta) \\
& =r(z)+r_{1}\left|z_{1}\right|+\left|z^{\prime}\right|^{2} \\
& \approx\left|z_{1}\right|+\left|z^{\prime}\right|^{2}+O\left(\left|z_{1}\right|^{2}+\left|z_{1}\right|\left|z^{\prime}\right|+\left|z^{\prime}\right|^{3}\right) .
\end{aligned}
$$

Therefore, from (3-10), there exist $C>1$ (by shrinking $\delta>0$ if necessary) such that

$$
|X(\phi(z), \eta)-X(\phi(\zeta), \eta)| \geq \frac{1}{C}|X(z, \zeta)|, \quad|z|<\delta .
$$

Note that if $|X(\phi(\zeta), \eta)|<\frac{1}{2 C}|X(z, \zeta)|$, the triangular inequality yields

$$
|X(\phi(z), \eta)| \gtrsim[|X(\phi(\zeta), \eta)|+|X(z, \zeta)|], \quad|z|<\delta .
$$

This inequality also holds when

$$
|X(\phi(\zeta), \eta)| \geq \frac{1}{2 C}|X(z, \zeta)|,
$$

since $|X(\phi(z), \eta)|$ has a minimum at $\zeta$. The constants involved depend continuously on $\eta$ throughout the calculations, and thus, by shrinking $\delta>0$ again if necessary, there are $C>0$ and $\delta>0$ such that

$$
|X(\phi(z), \eta)| \geq C[|X(\phi(\zeta), \eta)|+|X(z, \zeta)|]
$$

for all $(z, \eta) \in K_{\delta}$.

Since

$$
|X(z, \zeta)| \approx\left|z_{1}\right|+\left|z^{\prime}\right|^{2}+O\left(\left|z_{1}\right|^{2}+\left|z_{1}\right|\left|z^{\prime}\right|+\left|z^{\prime}\right|^{3}\right),
$$

the converse inequality follows from (3-9).

We use the same notation as in the proof of Proposition 3.1, and let

$$
r_{222}=\frac{\partial^{3} r(\zeta)}{\partial z_{2}^{3}}, \quad r_{22 \overline{2}}=\frac{\partial^{3} r(\zeta)}{\partial z_{2}^{2} \partial \bar{z}_{2}}
$$

We use the following lemma to prove the jump phenomenon when $C_{\phi}$ is not bounded on $A_{\alpha}^{p}(D)$.

Lemma 3.3. Let $\zeta=(0, \ldots, 0) \in \partial D$ with

$$
v(\zeta)=(1,0, \ldots, 0)
$$


and let $R$ be a holomorphic polynomial

$$
R\left(z_{1}, z_{2}\right)=r_{1} z_{1}+\left(r_{12}+r_{1 \overline{2}}\right) z_{1} z_{2}+\frac{\left(r_{22}+r_{2 \overline{2}}\right)}{2} z_{2}^{2}+\frac{\left(r_{222}+3 r_{22 \overline{2}}\right)}{6} z_{2}^{3} .
$$

Let $a \in \mathbb{C}, b \in \mathbb{R}$, and

$$
g(z)=\left(1+a z_{2}\right) R\left(z_{1}, z_{2}\right)+i b z_{2}^{3}+O\left(\left|z_{1}\right|^{2}+\left|z_{2}\right|^{4}+\left|z^{\prime \prime}\right|^{2}\right) .
$$

Then, for $\alpha \geq-1$, there is $C>0$ such that, for all $\delta>0$,

$$
V_{\alpha+1 / 4}(\{z \in D:|g(z)| \leq \delta\}) \geq C \delta^{n+\alpha+1} .
$$

Proof. It suffices to prove for $\delta>0$ small, and hence we assume $\delta>0$ is sufficiently small. For the rest of proof we assume

$$
z^{\prime}=\left(z_{2}, z^{\prime \prime}\right) \in A_{\delta}:=\left\{\left(z_{2}, z^{\prime \prime}\right) \in \mathbb{C}^{n-1}: x_{2}^{4}+y_{2}^{2}+\left|z^{\prime \prime}\right|^{2} \leq \delta\right\} .
$$

From the fact that $v(\zeta)=(1,0, \ldots, 0)$, there are constants $p_{j} \in \mathbb{R}$ for $1 \leq j \leq 5$ such that

$$
\begin{aligned}
r\left(z_{1}, z_{2}, z^{\prime \prime}\right)=r_{1} x_{1}+p_{1} x_{1} x_{2}+p_{2} y_{1} x_{2} & +p_{3} x_{2}^{2}+p_{4} x_{2}^{3}+p_{5} x_{2} y_{2} \\
& +O\left(x_{1}^{2}+y_{1}^{2}+y_{2}^{2}+x_{2}^{4}+\left|z^{\prime \prime}\right|^{2}\right) .
\end{aligned}
$$

Also, there are $q_{j} \in \mathbb{R}$ for $1 \leq j \leq 5$ such that

(3-15) $\operatorname{Im}\left[R\left(z_{1}+i y_{1}, z_{2}\right)+i b z_{2}^{3}\right]=r_{1} y_{1}+q_{1} y_{1} x_{2}+q_{1} x_{1} x_{2}+q_{3} x_{2} y_{2}+q_{4} x_{2}^{2}+q_{5} x_{2}^{3}$

$$
+O\left(x_{1}^{2}+y_{1}^{2}+y_{2}^{2}+x_{2}^{4}\right),
$$

since $\left|z_{1}\right|\left|y_{2}\right|+\left|x_{2}^{2} y_{2}\right|=O\left(x_{1}^{2}+y_{1}^{2}+y_{2}^{2}+x_{2}^{4}\right)$.

Taking $\delta>0$ sufficiently small if necessary, we may assume $r_{1}+p_{1} x_{2} \geq r_{1} / 2$ and $r_{1}+q_{1} x_{2} \geq r_{1} / 2$. Let $(u, v)=\left(u\left(z_{2}\right), v\left(z_{2}\right)\right) \in \mathbb{R}^{2}$ be the solution of the equations

$$
\begin{aligned}
& 0=\left(r_{1}+p_{1} x_{2}\right) u+p_{2} x_{2} v+p_{3} x_{2}^{2}+p_{4} x_{2}^{3}+p_{5} x_{2} y_{2}, \\
& 0=\left(r_{1}+q_{1} x_{2}\right) v+q_{2} x_{2} u+q_{3} x_{2} y_{2}+q_{4} x_{2}^{2}+q_{5} x_{2}^{3} .
\end{aligned}
$$

Since $z^{\prime} \in A_{\delta}$, the solution $(u, v)$ always exists and satisfies

$$
|u|+|v| \lesssim \delta^{1 / 2}
$$

Hence, by (3-14) and (3-15), we have

$$
r\left(u+i v, z_{2}, z^{\prime \prime}\right)=O(\delta), \quad \operatorname{Im}\left[R\left(u+i v, z_{2}\right)+i b z_{2}^{3}\right]=O(\delta) .
$$

By (2-1) we have $r_{2 \overline{2}} \in \mathbb{R}$, and thus

$$
\operatorname{Re}\left[r_{22} z_{2}\left(z_{2}-\bar{z}_{2}\right)\right]=-2 r_{22} y_{2}^{2} .
$$


Therefore,

$2 \operatorname{Re}\left[R\left(z_{1}, z_{2}\right)\right]$

$$
\begin{aligned}
= & r\left(z_{1}, z_{2}, 0^{\prime \prime}\right)+2 \operatorname{Re}\left[r_{12} z_{1}\left(z_{2}-\bar{z}_{2}\right)\right]+\operatorname{Re}\left[r_{22} z_{2}\left(z_{2}-\bar{z}_{2}\right)\right] \\
& \quad+\operatorname{Re}\left[r_{22 \overline{2}} z_{2}^{2}\left(z_{2}-\bar{z}_{2}\right)\right]+O\left(\left|z_{1}\right|^{2}+\left|z_{2}\right|^{4}\right) \\
= & r\left(z_{1}, z_{2}, 0^{\prime \prime}\right)-4 y_{2} \operatorname{Im}\left[r_{12} z_{1}\right]-2 r_{2 \overline{2}} y_{2}^{2}-2 y_{2} \operatorname{Re}\left[r_{22 \overline{2}} z_{2}^{2}\right]+O\left(\left|z_{1}\right|^{2}+\left|z_{2}\right|^{4}\right) \\
= & r\left(z_{1}, z_{2}, 0^{\prime \prime}\right)+O\left(\left|z_{1}\right|^{2}+\left|z_{1} y_{2}\right|+y_{2}^{2}+\left|y_{2}\right|\left|z_{2}\right|^{2}+\left|z_{2}\right|^{4}\right) \\
= & r\left(z_{1}, z_{2}, 0^{\prime \prime}\right)+O\left(x_{1}^{2}+y_{1}^{2}+y_{2}^{2}+x_{2}^{4}\right) .
\end{aligned}
$$

Therefore, from (3-16) we have

$$
2 \operatorname{Re}\left[R\left(u+i v, z_{2}\right)\right]=O(\delta),
$$

and thus, from the second equation of (3-16), we have

$$
\left|R\left(u+i v, z_{2}\right)\right| \approx\left|\operatorname{Re}\left[R\left(u+i v, z_{2}\right)\right]\right|+\left|\operatorname{Im}\left[R\left(u+i v, z_{2}\right)\right]\right|=O(\delta) .
$$

From these estimates we then have

$$
\begin{aligned}
\left|g\left(u+i v, z^{\prime}\right)\right| \lesssim & \left|\operatorname{Re}\left[R\left(u+i v, z_{2}\right)\right]\right|+\left|z_{2}\right|\left|R\left(u+i v, z_{2}\right)\right| \\
& \quad+\left|\operatorname{Im}\left[R\left(u+i v, z_{2}\right)+i b z_{2}^{3}\right]\right|+O\left(|u+i v|^{2}+x_{2}^{4}+y_{2}^{2}+\left|z^{\prime \prime}\right|^{2}\right) \\
= & O(\delta) .
\end{aligned}
$$

Since $\partial g(\zeta) / \partial z_{1}=r_{1}$, by taking $\delta$ sufficiently small if necessary, we have

$$
z_{1}=u\left(z_{2}\right)+i v\left(z_{2}\right)+O(\delta) \Longrightarrow|g(z)| \lesssim \delta .
$$

Let

$$
B_{\delta}^{C}\left(z_{2}\right):=\left\{z_{1}: u\left(z_{2}\right)+C \delta \leq x_{1} \leq u\left(z_{2}\right)+2 C \delta, v\left(z_{2}\right) \leq y_{1} \leq v\left(z_{2}\right)+\delta\right\}
$$

and

$$
\Lambda_{\delta}^{C}=\left\{z: z^{\prime} \in A_{\delta}, z_{1} \in B_{\delta}^{C}\left(z_{2}\right)\right\} .
$$

Then, by (3-14), there is $C>0$ such that, for all $z \in \Lambda_{\delta}^{C}$, we have

$$
r(z) \approx \delta
$$

and from (3-17), for all $z \in \Lambda_{\delta}^{C}$, we have

$$
\left|g\left(z_{1}, z_{2}, z^{\prime \prime}\right)\right| \lesssim \delta
$$

Therefore, there are constants $c, C>0$ such that

$$
V_{\alpha+1 / 4}(\{z \in D:|g(z)| \leq \delta\}) \geq V_{\alpha+1 / 4}\left(\Lambda_{c \delta}^{C}\right) \gtrsim \delta^{\alpha+1 / 4} V\left(\Lambda_{c \delta}^{C}\right) .
$$


Since $B_{\delta}^{C}\left(z_{2}\right)$ is a rectangle with area $C \delta^{2}$ for a fixed $z_{2}$, from the definition of $A_{\delta}$ in (3-13) we have

$$
V_{\alpha+1 / 4}(\{z \in D:|g(z)| \leq \delta\}) \gtrsim \delta^{\alpha+1 / 4} V\left(\Lambda_{c \delta}^{C}\right) \approx \delta^{\alpha+n+1} .
$$

The proof is complete, since the constants suppressed in the inequalities throughout our calculations are independent of $\delta$.

\section{Proof of Theorem 1.1}

First, we prove the last statement, the jump phenomenon, assuming the equivalence of (1), (2), and (3).

Let $0<\epsilon<\frac{1}{4}$ and suppose

$$
C_{\phi}: A_{\alpha}^{p}(D) \rightarrow A_{\alpha+\epsilon}^{p}(D)
$$

is bounded. Then

$$
C_{\phi}: A_{\alpha}^{p}(D) \rightarrow A_{\alpha+1 / 4}^{p}(D)
$$

is compact, since the inclusion the map $I: A_{\alpha+\epsilon}^{p}(D) \hookrightarrow A_{\alpha+1 / 4}^{p}(D)$ is compact. Thus, from the equivalence of (1) and (2) we conclude the boundedness of

$$
C_{\phi}: A_{\alpha}^{p}(D) \rightarrow A_{\alpha}^{p}(D) .
$$

To prove the equivalence of (1), (2), and (3), note that (1) $\Longrightarrow(2)$ is trivial since the inclusion map $I: A_{\alpha}^{p}(D) \hookrightarrow A_{\alpha+1 / 4}^{p}(D)$ is compact. Thus, it suffices to show that (2) $\Longrightarrow$ (3) and (3) $\Longrightarrow$ (1). First (3) $\Longrightarrow$ (1) follows from the following theorem.

Theorem 4.1. Let $0<p<\infty$ and $\alpha \geq-1$. Let $\phi: D \rightarrow D$ be a holomorphic map with $\phi \in C^{4}(\bar{D})$. If $Q_{\phi}(\zeta)<1$ on $\phi^{-1}(\partial D)$, then $C_{\phi}$ is bounded on $A_{\alpha}^{p}(D)$.

Proof. Let $\mu=\sigma \circ \phi^{-1}$ and $\mu_{\alpha}=V_{\alpha} \circ \phi^{-1}$ for $\alpha>-1$. By Corollary 2.2, it suffices to show that there exist $\delta_{0}>0$ and $M>0$ such that, for all $\eta \in \partial D$ and $0<\delta<\delta_{0}$,

$$
\mu(\overline{C(\eta, \delta)}) \leq M \delta^{n}
$$

and

$$
\mu_{\alpha}(C(\eta, \delta)) \leq M \delta^{n+1+\alpha} .
$$

We may assume $\delta>0$ is sufficiently small, since, otherwise, (4-1) and (4-2) hold trivially. Note that $\phi(D) \cap \partial D=\varnothing$ since $\phi$ is a holomorphic self-map of $D$. Thus $\phi(\bar{D}) \cap[\partial D \backslash V]=\varnothing$ for any neighborhood $V \subset \partial D$ of $\partial D \cap \phi(\partial D)$. By (2-4), with $W_{\delta}$ as defined right before Lemma 3.2, it suffices to show that there are constants $\delta_{1}>0$ and $\delta_{2}>0$ such that (4-1) and (4-2) hold for all $\delta<\delta_{1}$ and $\eta \in W_{\delta_{2}}$. Choose $\delta_{1}$ and $\delta_{2}$ small so that Lemma 3.2 holds with $\delta=\delta_{0}:=\left(\delta_{1}+\delta_{2}\right)$, and let $C>1$ be the corresponding constant in Lemma 3.2. 
For $\eta \in W_{\delta_{2}}$, let $O_{j}$ be any component of $\phi^{-1}\left(\mathscr{C}\left(\eta, \delta_{0}\right)\right)$ which also intersects with $\phi^{-1}\left(\mathscr{C}\left(\eta, \delta_{0} / 2 C\right)\right)$. Let $\zeta_{j} \in \overline{O_{j}}$ be a point such that

$$
\min \left\{|X(\phi(w), \eta)|: w \in \overline{O_{j}}\right\}=\left|X\left(\phi\left(\zeta_{j}\right), \eta\right)\right| .
$$

Since $\left|X\left(\phi\left(\zeta_{j}\right), \eta\right)\right| \leq \delta_{0} / 2 C$, by (3-8) we have

$$
\phi\left(\mathscr{C}\left(\zeta_{j}, \delta_{0} / 2 C\right)\right) \subset \mathscr{C}\left(\eta, \delta_{0}\right) .
$$

Therefore, $\mathscr{C}\left(\zeta_{j}, \delta_{0} / 2 C\right) \subset O_{j}$, since $O_{j}$ is a component which contains $\zeta_{j}$. This implies that the number of components $O_{j}$ has an upper bound $M<\infty$ independent of $\eta$, since

$$
M \delta_{0}^{n+1+\alpha} \approx \sum_{j=1}^{M} V_{\alpha}\left(\mathscr{C}\left(\zeta_{j}, \delta_{0} / 2 C\right)\right) \leq V_{\alpha}\left(\phi^{-1}\left(\mathscr{C}\left(\eta, \delta_{0}\right)\right)\right) \lesssim 1 .
$$

Now fix such a component $O_{j}$ as above. Then, by Lemma 3.2,

$$
O_{j} \cap \phi^{-1}(\mathscr{C}(\eta, \delta)) \subset \mathscr{C}\left(\zeta_{j}, C \delta\right)
$$

for all $\delta<\delta_{0}$.

Then, (4-1) and (4-2) follows immediately since the number of components has a uniform upper bound $M$.

Next, $(2) \Longrightarrow$ (3) follows from the following theorem together with the Carleson measure criteria, Corollary 2.2.

Theorem 4.2. Let $\phi: D \rightarrow D$ be a holomorphic map with $\phi \in C^{4}(\bar{D})$. Suppose $\zeta, \eta=\phi(\zeta) \in \partial D$ and $Q_{\phi}(\zeta)=1$. Then there is $C>0$ such that, for all $\delta>0$,

$$
V_{\alpha+1 / 4}\left(\phi^{-1}(\mathscr{C}(\eta, \delta))\right) \geq C V_{\alpha}(\mathscr{C}(\eta, \delta))
$$

and

$$
V_{-3 / 4} \circ \phi^{-1}(\overline{(\mathscr{C}(\eta, \delta)}) \geq C \sigma(\overline{\mathscr{C}(\eta, \delta)} \cap \partial D) .
$$

Proof. For $z \in \mathbb{C}^{n}$, let $z=\left(z_{1}, \ldots, z_{n}\right)=\left(z_{1}, z^{\prime}\right)=\left(z_{1}, z_{2}, z^{\prime \prime}\right)$. Near $(\zeta, \eta) \in$ $\partial D \times \partial D$, we choose the same coordinates as in the proof of Proposition 3.1 so that

$$
\zeta=\eta=(0, \ldots, 0), \quad v(\zeta)=v(\eta)=(1,0, \ldots, 0) .
$$

By change of coordinates in $z^{\prime}$ variables if necessary, we may assume $Q_{\phi}(\zeta)=1$ for $\tau=(0,1,0, \ldots, 0)$, that is,

$$
\left|\frac{\mathscr{D}_{\tau \tau}^{2} \phi_{\eta}(\zeta)}{\mathscr{D}_{\mathcal{V}(\zeta)} \phi_{\eta}(\zeta)}-\frac{\mathscr{D}_{\tau \tau}^{2} r(\zeta)}{|\partial r(\zeta)|}\right| \cdot \frac{|\partial r(\zeta)|}{\left|\mathscr{D}_{\tau \bar{\tau}}^{2} r(\zeta)\right|}=1 \quad(\tau=(0,1,0, \ldots, 0)) .
$$

Since this relation is invariant under rotation in the $z_{2}$ variable, we may assume

$$
\frac{\mathscr{D}_{\tau \tau}^{2} \phi_{\eta}(\zeta)}{\mathscr{D}_{\mathcal{V}(\zeta)} \phi_{\eta}(\zeta)}-\frac{r_{22}}{r_{1}}=\frac{r_{2 \overline{2}}}{r_{1}}
$$


By (1) and (2) of Proposition 3.1, we have

$$
\phi_{\eta}(z)=a_{1} z_{1}+\sum_{j=2}^{n} a_{2 j} z_{2} z_{j}+a_{32} z_{2}^{3}+O\left(\left|z_{1}\right|^{2}+\left|z_{2}\right|^{4}+\left|z^{\prime \prime}\right|^{2}\right)
$$

with $a_{1}>0$. Therefore, the condition $Q_{\phi}(\zeta)=1$ is equivalent to

$$
\frac{2 a_{22}}{a_{1}}-\frac{r_{22}}{r_{1}}=\frac{r_{2 \overline{2}}}{r_{1}} \text {. }
$$

Let $R\left(z_{1}, z_{2}\right)$ be as in (3-12). Then, by (4-3) and (4-4), we get

$$
\begin{aligned}
\phi_{\eta}(z)=\frac{a_{1}}{r_{1}}\left(1+A z_{2}\right) R\left(z_{1}, z_{2}\right)+B z_{2}^{3} & \\
& +\sum_{j=3}^{n} a_{2 j} z_{2} z_{j}+O\left(\left|z_{1}\right|^{2}+\left|z_{2}\right|^{4}+\left|z_{1}\right|\left|z_{3}\right|^{2}+\sum_{j=4}^{n}\left|z_{j}\right|^{2}\right),
\end{aligned}
$$

where

$$
A=\frac{a_{12}}{a_{1}}-\frac{\left(r_{22}+r_{22}\right) a_{12}}{2 r_{1}}, \quad B=a_{32}-\frac{\left(r_{222}+3 r_{22 \overline{2}}\right) a_{1}}{6 r_{1}}-A \frac{\left(r_{22}+r_{22}\right) a_{1}}{2 r_{1}} .
$$

Then, by Lemma 3.3, to complete the proof it suffices to show that

$$
\operatorname{Re} B=0, \quad a_{2 j}=0 \quad(j=3, \ldots, n) .
$$

Since $v(\zeta)=(1,0, \ldots, 0)$, for $(s, t) \in \mathbb{R}^{2}$ we have

$$
r\left(s, t, t e^{i \theta}, 0, \ldots, 0\right)=2 r_{1} s+O\left(s^{2}+t^{2}\right) .
$$

Thus, for each $\theta, t \in \mathbb{R}$, there is $s \in \mathbb{R}$ with $|s| \lesssim t^{2}$ such that $\operatorname{Re}[R(s, t)]=$ $r\left(s, t, t e^{i \theta}, 0, \ldots, 0\right)=0$.

Since $\operatorname{Re} \phi_{\eta}\left(s, t, t e^{i \theta}, 0, \ldots, 0\right) \geq 0$ by (2-3), we get

$$
\begin{aligned}
0 & \leq \operatorname{Re} \phi_{\eta}\left(s, t, t e^{i \theta}, 0, \ldots, 0\right) \\
& =\operatorname{Re}\left[\frac{a_{1}}{r_{1}}(1+A t) R(s, t)+B t^{3}+a_{23} t^{2} e^{i \theta}\right]+O\left(s^{2}+t^{4}\right) \\
& =\operatorname{Re}\left[\frac{a_{1}}{r_{1}} A t R(s, t)+B t^{3}+a_{23} t^{2} e^{i \theta}\right]+O\left(s^{2}+t^{4}\right) \\
& =\operatorname{Re}\left[B t^{3}+a_{23} t^{2} e^{i \theta}\right]+O\left(s^{2}+t^{4}\right)
\end{aligned}
$$

for all $\theta$. This implies $a_{23}=0$, and, with the same argument, we get

$$
a_{2 j}=0 \quad(j=3, \ldots, n) .
$$

Also, note that $r\left(s, \pm t, 0^{\prime \prime}\right)=2 r_{1} s+O\left(s^{2}+t^{2}\right)$ which implies that for each $\pm t$ 
there is $s=s( \pm t)$ such that $r\left(s, \pm t, 0^{\prime \prime}\right)=0$ with $|s( \pm t)| \lesssim t^{2}$. Then, by (2-3), with $s=s( \pm t)$ we have

$$
\begin{aligned}
0 \leq \operatorname{Re} \phi_{\eta}\left(s, \pm t, 0^{\prime \prime}\right) & =\frac{a_{1}}{r_{1}} \operatorname{Re}[R(s, \pm t)] \pm t^{3} \operatorname{Re} B+O\left(t|\operatorname{Im}[R(s, \pm t)]|+t^{4}\right) \\
& = \pm t^{3} \operatorname{Re} B+O\left(t^{4}\right)
\end{aligned}
$$

Therefore, we get $\operatorname{Re} B=0$ and the proof is complete.

\section{Acknowledgement}

Part of this research was performed during the first author's visit to University of California at Irvine. He thanks the Mathematics Department of University California at Irvine for its hospitality and support.

\section{References}

[Cima et al. 1984] J. A. Cima, C. S. Stanton, and W. R. Wogen, "On boundedness of composition operators on $H^{2}\left(B_{2}\right)$ ", Proc. Amer. Math. Soc. 91:2 (1984), 217-222. MR 85j:47030 Zbl 0546.47015

[Fefferman 1974] C. Fefferman, "The Bergman kernel and biholomorphic mappings of pseudoconvex domains”, Invent. Math. 26 (1974), 1-65. MR 50 \#2562 Zbl 0289.32012

[Hörmander 1967] L. Hörmander, " $L^{p}$ estimates for (pluri-) subharmonic functions", Math. Scand. 20 (1967), 65-78. MR 38 \#2323 Zbl 0156.12201

[Koo and Park 2010] H. Koo and I. Park, "Composition operators on holomorphic Sobolev spaces in $B_{n}$ ”, J. Math. Anal. Appl. 369:1 (2010), 232-244. MR 2011e:47045 Zbl 1191.47031

[Koo and Smith 2007] H. Koo and W. Smith, "Composition operators induced by smooth self-maps of the unit ball in $\mathscr{C}^{N ",}$, J. Math. Anal. Appl. 329:1 (2007), 617-633. MR 2008e:47059 Zbl 1115.32004

[Koo and Wang 2010] H. Koo and M. Wang, "Revisit to a theorem of Wogen", pp. 355-363 in Topics in operator theory, 1: Operators, matrices and analytic functions, edited by J. A. Ball et al., Oper. Theory Adv. Appl. 202, Birkhäuser, Basel, 2010. MR 2012a:47057 Zbl 1221.47044

[Krantz 2001] S. G. Krantz, Function theory of several complex variables, AMS Chelsea, Providence, RI, 2001. MR 2002e:32001 Zbl 1087.32001

[Krantz and Li 1994] S. G. Krantz and S.-Y. Li, "A note on Hardy spaces and functions of bounded mean oscillation on domains in $\breve{C}^{n} "$, Michigan Math. J. 41:1 (1994), 51-71. MR 95f:32008 Zbl 0802.32013

[Krantz and Li 1995a] S. G. Krantz and S.-Y. Li, "Duality theorems for Hardy and Bergman spaces on convex domains of finite type in $\mathscr{C}^{n} "$, Ann. Inst. Fourier (Grenoble) 45:5 (1995), 1305-1327. MR 96m:32002 Zbl 0835.32004

[Krantz and Li 1995b] S. G. Krantz and S.-Y. Li, "On decomposition theorems for Hardy spaces on domains in $\mathscr{C}^{n}$ and applications", J. Fourier Anal. Appl. 2:1 (1995), 65-107. MR 96m:32003 Zbl 0886.32003

[MacCluer 1984] B. D. MacCluer, "Spectra of compact composition operators on $H^{p}\left(B_{N}\right)$ ", Analysis 4:1-2 (1984), 87-103. MR 86e:47038 Zbl 0582.32009

[Phong and Stein 1977] D. H. Phong and E. M. Stein, "Estimates for the Bergman and Szegö projections on strongly pseudo-convex domains", Duke Math. J. 44:3 (1977), 695-704. MR 56 \#8916 Zbl 0392.32014 
[Wogen 1988] W. R. Wogen, "The smooth mappings which preserve the Hardy space $H^{2}\left(B_{n}\right)$ ", pp. 249-263 in Contributions to operator theory and its applications (Mesa, AZ, 1987), edited by I. Gohberg et al., Oper. Theory Adv. Appl. 35, Birkhäuser, Basel, 1988. MR 90k:32018 Zbl 0685.46029

Received September 26, 2012. Revised May 19, 2013.

HYUNGWOON KOO

DEPARTMENT OF MATHEMATICS

KOREA UNIVERSITY

SEOUL 136-713

SOUTH KOREA

koohw@korea.ac.kr

\section{SONG-YING LI}

DEPARTMENT OF MATHEMATICS

UNIVERSITY OF CALIFORNIA, IRVINE

IRVINE, CA 92697

UNITED STATES

sli@math.uci.edu

and

SCHOOL OF MATHEMATICS AND COMPUTER SCIENCES

FUJIAN NORMAL UNIVERSITY

FUJIAN

CHINA 


\title{
PACIFIC JOURNAL OF MATHEMATICS
}

\author{
msp.org/pjm
}

Founded in 1951 by E. F. Beckenbach (1906-1982) and F. Wolf (1904-1989)

\section{EDITORS}

V. S. Varadarajan (Managing Editor)

Department of Mathematics

University of California

Los Angeles, CA 90095-1555

pacific@math.ucla.edu

Paul Balmer

Department of Mathematics

University of California

Los Angeles, CA 90095-1555

balmer@math.ucla.edu

Daryl Cooper

Department of Mathematics

University of California

Santa Barbara, CA 93106-3080 cooper@math.ucsb.edu

Jiang-Hua $\mathrm{Lu}$

Department of Mathematics

Pokfulam Rd., Hong Kong jhlu@maths.hku.hk
The University of Hong Kong

Don Blasius

Department of Mathematics University of California

Los Angeles, CA 90095-1555

blasius@math.ucla.edu

Robert Finn

Department of Mathematics Stanford University

Stanford, CA 94305-2125

finn@math.stanford.edu

Sorin Popa

Department of Mathematics

University of California

Los Angeles, CA 90095-1555 popa@math.ucla.edu

Paul Yang

Department of Mathematics Princeton University

Princeton NJ 08544-1000

yang@math.princeton.edu

\section{PRODUCTION}

Silvio Levy, Scientific Editor, production@msp.org

\section{SUPPORTING INSTITUTIONS}

ACADEMIA SINICA, TAIPEI

CALIFORNIA INST. OF TECHNOLOGY

INST. DE MATEMÁTICA PURA E APLICADA

KEIO UNIVERSITY

MATH. SCIENCES RESEARCH INSTITUTE

NEW MEXICO STATE UNIV.

OREGON STATE UNIV.

\author{
STANFORD UNIVERSITY \\ UNIV. OF BRITISH COLUMBIA \\ UNIV. OF CALIFORNIA, BERKELEY \\ UNIV. OF CALIFORNIA, DAVIS \\ UNIV. OF CALIFORNIA, LOS ANGELES \\ UNIV. OF CALIFORNIA, RIVERSIDE \\ UNIV. OF CALIFORNIA, SAN DIEGO \\ UNIV. OF CALIF., SANTA BARBARA
}

\author{
Vyjayanthi Chari \\ Department of Mathematics \\ University of California \\ Riverside, CA 92521-0135 \\ chari@math.ucr.edu \\ Kefeng Liu \\ Department of Mathematics \\ University of California \\ Los Angeles, CA 90095-1555 \\ liu@math.ucla.edu \\ Jie Qing \\ Department of Mathematics \\ University of California \\ Santa Cruz, CA 95064 \\ qing@cats.ucsc.edu
}

These supporting institutions contribute to the cost of publication of this Journal, but they are not owners or publishers and have no responsibility for its contents or policies.

See inside back cover or msp.org/pjm for submission instructions.

The subscription price for 2014 is US \$410/year for the electronic version, and \$535/year for print and electronic.

Subscriptions, requests for back issues and changes of subscribers address should be sent to Pacific Journal of Mathematics, P.O. Box 4163, Berkeley, CA 94704-0163, U.S.A. The Pacific Journal of Mathematics is indexed by Mathematical Reviews, Zentralblatt MATH, PASCAL CNRS Index, Referativnyi Zhurnal, Current Mathematical Publications and Web of Knowledge (Science Citation Index).

The Pacific Journal of Mathematics (ISSN 0030-8730) at the University of California, c/o Department of Mathematics, 798 Evans Hall \#3840, Berkeley, CA 94720-3840, is published twelve times a year. Periodical rate postage paid at Berkeley, CA 94704, and additional mailing offices. POSTMASTER: send address changes to Pacific Journal of Mathematics, P.O. Box 4163, Berkeley, CA 94704-0163.

PJM peer review and production are managed by EditFLOW ${ }^{\circledR}$ from Mathematical Sciences Publishers.

\section{PUBLISHED BY}

mathematical sciences publishers

nonprofit scientific publishing

http://msp.org/

(C) 2014 Mathematical Sciences Publishers 


\section{PACIFIC JOURNAL OF MATHEMATICS}

Volume $268 \quad$ No. $1 \quad$ March 2014

AlEXANDRE PAIVA BARRETO

A transport inequality on the sphere obtained by mass transport

DARIO CORDERO-ERAUSQUIN

A cohomological injectivity result for the residual automorphic spectrum of $\mathrm{GL}_{n}$

HARALD GROBNER

Gradient estimates and entropy formulae of porous medium and fast diffusion equations for the Witten Laplacian

GUANGYUE HUANG and HAIZHONG LI

Controlled connectivity for semidirect products acting on locally finite trees

KeITH JoNES

An indispensable classification of monomial curves in $\mathbb{A}^{4}(\mathbb{k})$

ANARGYROS KATSABEKIS and IGNACIO OJEDA

Contracting an axially symmetric torus by its harmonic mean curvature

CHRISTOPHER KIM

Composition operators on strictly pseudoconvex domains with smooth symbol

HYUNGWOON KOO and SONG-YING LI

The Alexandrov problem in a quotient space of $\mathbb{U}^{2} \times \mathbb{R}$

AnA Menezes

Twisted quantum Drinfeld Hecke algebras

DEEPAK NAIDU

$L^{p}$ harmonic 1-forms and first eigenvalue of a stable minimal hypersurface

KEOMKYO SEO

Reconstruction from Koszul homology and applications to module and derived categories

RYO TAKAHASHI

A virtual Kawasaki-Riemann-Roch formula 